Research Paper

\title{
Krüppel-Like Factor 5 Promotes Epithelial Proliferation and DNA Damage Repair in the Intestine of Irradiated Mice
}

\author{
Ming Li1,2*, Yuan $\mathrm{Gu}^{1,2 *}$, Yan-Chao Ma ${ }^{3,4}$, Zeng-Fu Shang, ${ }^{1,2}$, Chang Wang, ${ }^{1,2}$, Fen-Ju Liu ${ }^{1,2}$, Jian-Ping Cao ${ }^{1,2}$, \\ Hua-Jing $W^{5}{ }^{5}$, Xue-Guang Zhang ${ }^{3,4} \bowtie$ \\ 1. School of Radiation Medicine and Protection, Medical College of Soochow University, Suzhou, Jiangsu 215123, China \\ 2. Collaborative Innovation Center of Radiation Medicine of Jiangsu Higher Education Institutions, Suzhou, Jiangsu 215123, China \\ 3. Institute of Medical Biotechnology, Medical College of Soochow University, Suzhou, Jiangsu 215007, China \\ 4. Jiangsu Stem Cell Key Laboratory, Medical College of Soochow University, Suzhou, Jiangsu 215007, China \\ 5. Key Laboratory of Obstetric, Gynecologic and Pediatric Diseases and Birth Defects of the Ministry of Education, West China Institute of Women and Chil- \\ dren's Health, and Department of Pediatrics, Huaxi Second University Hospital, Sichuan University, Chengdu, Sichuan 610041, China. \\ *These authors contributed equally to this work. \\ $\square$ Corresponding author: Xue-Guang Zhang, Ph.D., Institute of Medical Biotechnology and Jiangsu Stem Cell Key Laboratory, Medical College of Soochow \\ University, Suzhou, Jiangsu 215007, China; E-mail: xueguangzh@126.com; Tel: +86-0512-65732002; Fax: +86-0512-65884830.
}

(C) 2015 Ivyspring International Publisher. Reproduction is permitted for personal, noncommercial use, provided that the article is in whole, unmodified, and properly cited. See http:/ /ivyspring.com/terms for terms and conditions.

Received: 2015.08.04; Accepted: 2015.11.01; Published: 2015.12.02

\begin{abstract}
BACKGROUND \& AIMS: High doses of radiation induce severe DNA damage in intestinal epithelial cells, especially crypt cells, and cause intestinal injury, but the underlying molecular mechanisms remain unclear. Krüppel-like factor 5 (KLF5), a zinc finger-containing transcription factor, is induced by various stress stimuli and is involved in cell proliferation and survival. The role of KLF5 in radiation-induced intestinal injury was investigated here.

METHODS: Wild type mice were treated with 8 or 15 Gy total body irradiation (TBI). KLF5 content and cellular localization in the small intestines of irradiated mice were detected by Western blot and immunohistochemical analysis. Mice with intestinal-specific knockdown of KLF5 (Vil-Cre; Klf5 fl/+ mice) were generated and their response to radiation was compared with controls. Morphological changes were determined by hematoxylin and eosin staining. Proliferation was examined by Ki67 immunostaining. The molecular response of the small intestine after KLF5 knockdown was investigated using microarrays.

RESULTS: KLF5 expression correlated with the progression of intestinal damage. Decreased levels of KLF5 in the gut were associated with increased damage to the intestinal mucosa and reduced epithelial proliferation after TBI. Our microarray data disclosed that KLF5 knockdown down-regulated genes related to DNA damage repair pathways such as nucleotide excision repair, mismatch repair, non-homologous end joining and the Fanconi anemia pathway, which may suggest a novel function of KLF5.

CONCLUSIONS: Our study illustrates that KLF5 may modulate DNA repair pathways to prevent intestinal injury induced by TBI. KLF5 signaling provides a novel field for identification of potential therapeutic targets for the treatment of radiation-induced intestinal damage.
\end{abstract}

Key words: Krüppel-like factor 5; ionizing radiation; intestinal injury; mouse

\section{Introduction}

The small intestine is the major site of nutrient digestion and absorption and serves as a vital barrier against diverse harmful and immunogenic substances within the mammalian intestinal lumen. Gut integrity is important for its function. However, the small intestinal epithelium is inherently sensitive to irradia- 
tion due to rapid cell turnover [1-3]. High doses of radiation cause apoptosis in crypts, shortening of villi, and even loss of intestinal mucosa. Radiation-induced intestinal injuries are common side-effects of radiotherapy for abdominal or pelvic cancer. Additionally, accidental radiation exposure may alter the small intestine structurally and functionally. Morphological changes of the intestinal mucosa after ionizing radiation have been well documented [4-6], but molecular events that regulate intestinal epithelial cells radiosensitivity and radiation-induced intestinal injuries are not fully understood.

Ionizing radiation can induce DNA damage, producing single- and double-strand DNA breaks [7]. Mammals have evolved several DNA repair pathways to maintain genomic stability, including mismatch repair (MMR), base excision repair (BER), nucleotide excision repair (NER), homologous recombination repair (HR), and non-homologous end joining (NHEJ). In addition, the Fanconi anemia pathway promotes HR. Previous studies confirm that DNA repair protein deficiencies such as ataxia telangiectasia mutated (ATM) [8], 53BP1 [9], poly ADP-ribose polymerase-1 (PARP-1) [10], and NHEJ factors RAD50, MRE11, or DNA-PK [11] generally elevate intestinal sensitivity and promote intestinal injury and subsequent lethality. Moreover, deficiencies in MMR proteins MSH2 [12], MLH1, or PMS2 [13] lead to enhanced carcinogenesis, probably by increasing stem cell survival and mutation rates. Recent studies have shown that crypt base columnar stem cells $(\mathrm{CBCs})$ are radiation resistant, repairing DNA damage by HR [14]. However, there is still much to be discovered about DNA repair mechanisms in radiation-induced intestinal injury.

Krüppel-like factor 5 (KLF5, also named BTEB2 [15] and intestine-enriched KLF, IKLF) is an evolutionarily conserved zinc finger-containing transcription factor that belongs to the KLF family. KLF5 regulates a diverse array of important target genes, including those encoding as cyclin D1 [16], cyclin B1 [17], fibroblast growth factor binding protein 1 (FGF-BP) [18], epithelial growth factor receptor (EGFR) [19] and survivin [20], and plays important roles in multiple cellular processes such as cell proliferation, differentiation, migration, cell cycle, apoptosis and tissue development [21]. KLF5 is highly expressed in proliferating cells of intestinal crypts [22] and accumulated evidence from studies with genetically manipulated mice suggest that KLF5 is critical for regulating intestinal homeostasis and disease [23-28]. However, the role of KLF5 in radiation-induced intestinal injuries has not yet been investigated.
The best known function of KLF5 is its pro-proliferative role in different cell types. Mechanistically, KLF5 promotes cell proliferation by accelerating $G_{1} / S$ [29] and $G_{2} / M[17,30]$ cell cycle progression and up-regulating EGFR and MEK/ERK pathways [19]. In addition to stimulation of cell proliferation, KLF5 is also thought to be a stress response mediator. Several studies indicate that KLF5 is induced in response to various external stimuli and is involved in cell proliferation and survival. For example, KLF5 is up-regulated in response to DNA-damaging agents such as 5-fluorouracil and ultraviolet rays in HCT116 colon cancer cells. Cells deficient in KLF5 become more sensitive to 5-fluorouracil-induced apoptosis [31]. Additionally, KLF5 is induced by the bacterial membrane component lipopolysaccharide (LPS) through the MAPK pathway in IEC-6 rat intestinal epithelial cells [32]. KLF5 induction correlates with NF-KB induction and its downstream targets including interleukin-6 (IL-6) and tumor necrosis factor-a (TNF-a). In vivo studies conducted in mice treated with dextran sulfate sodium (DSS) [26] or infected with Citrobacter rodentium [25] confirm increased KLF5 expression in colon tissues. Reduced KLF5 enhances susceptibility to DSSand Citrobacter rodentium-induced damage. However, whether KLF5 is activated in response to ionizing radiation and plays any role in radiation-induced intestinal injuries remains elusive.

Here, we report studies of KLF5 expression in intestinal tissues of wild type (WT) mice after exposure to total body irradiation (TBI) at two different doses. Mice with intestinal-specific knockdown of the Klf5 gene (Vil-Cre; $\mathrm{Klfffl}^{f /+}$ ) were generated using the Cre-LoxP system and studied to explore the role of KLF5 in TBI-induced intestinal injuries. Our data indicate that KLF5 expression correlated with progression of intestinal damages. KLF5 knockdown increased intestinal mucosal damage induced by TBI. More importantly, our microarray data revealed that KLF5 knockdown down-regulated genes related to NER, MMR, NHEJ and the Fanconi anemia pathway, which may suggest a novel function of KLF5.

\section{Materials and Methods}

\section{Experimental animals}

WT male C57BL/6 mice (18-22g) were purchased from Shanghai SLAC Laboratory Animal Co., Ltd. (Shanghai, China). Mice with intestinal-specific knockdown of KLF5 were generated according to published methods [24]. Animals were housed in a pathogen-free barrier facility under controlled conditions, including a 12:12-h light-dark cycle, with ad libitum access to food and water. The study was car- 
ried out in accordance with protocols approved by the Animal Ethics Committee of Soochow University.

\section{Radiation procedure}

A single dose of 8 or 15 Gy TBI was delivered to mice using a 6-MeV electron linear accelerator (Clinac 2100EX; Varian Medical Systems, Palo Alto, CA, USA) at 2 Gy per minute.

\section{Protein extraction and Western blot}

Animals were killed at the indicated times after TBI and rapidly dissected. Small intestine tissues were flushed with phosphate-buffered saline (PBS). Protein extraction and Western blot were performed according to published methods [33]. Antibodies are listed in Supplementary methods and materials.

\section{Histology}

Small intestine tissues were isolated and fixed in $4 \%$ paraformaldehyde overnight and embedded in paraffin. Then, $3 \mu \mathrm{m}$-thick paraffin sections were stained with hematoxylin and eosin (H\&E) or appropriate staining reagents. Images of tissue sections were captured using an Olympus optical microscope (Olympus Corp., Tokyo, Japan).

\section{Immunohistochemical (IHC) analysis}

Localization of KLF5, Ki67 and Musashi-1 was examined by IHC according to published methods [33].

\section{Immunofluorescence (IF) assay}

The paraffin sections were deparaffinized and rehydrated. Heat-mediated antigen retrieval, blocking of non-specific binding sites and primary antibody incubation were performed as for IHC analysis. Fluorescein-labeled diluted secondary antibody was added and incubated at $37^{\circ} \mathrm{C}$ for $1 \mathrm{~h}$. Sections were counterstained with DAPI and imaged under a confocal laser scanning microscope (Leica Microsystems $\mathrm{GmbH}$, Wetzlar, Germany).

\section{Microarray analysis of gene expression}

Small intestines were isolated from control and Vil-Cre; $\mathrm{Klf5fl+}$ mice treated with 15 Gy TBI or no radiation. The microarray analysis protocol is provided in the Supplementary methods and materials.

\section{Quantitative real-time PCR (qRT-PCR) analy- sis}

Total RNA was isolated from tissues using Trizol reagent (Invitrogen, Carlsbad, CA, USA) according to the manufacturer's protocol. First-strand cDNA was synthesized from $3 \mu \mathrm{g}$ of total RNA using a PrimeScriptTM RT reagent Kit (Perfect Real Time) (TAKARA, Otsu, Japan). Primers are listed in Table
S1. QRT-PCR was performed using a SYBR ${ }^{\mathrm{B}}$ Premix Ex TaqTM (Tli RNaseH Plus) Real-Time PCR assay kit (TAKARA, Otsu, Japan) in a 7500 Real-Time PCR system (Applied Biosystems, Foster City, CA, USA). All samples were examined in triplicate.

\section{Statistical analysis}

Data are presented as means \pm SEM. SPSS 16.0 software (SPSS Inc., San Rafael, CA, USA) was used to perform statistical analysis. Equality of variances was tested by Levene's test. Data involving only two groups were analyzed using a Student's t-test. Data were assessed by one-way ANOVA when more than two groups were compared. Data were considered statistically significant if the $P$ value was less than 0.05 .

\section{Results}

\section{Alterations of KLF5 expression after radiation exposure}

Although small intestinal epithelia are sensitive to radiation exposure, intestinal structure can be renewed at a radiation dose of 8 Gy or less. At high doses of radiation ( $\geq 14 \mathrm{~Gy}$ ), mice die between 7 and 12 days due to irreparable intestinal damage concomitant with crypts loss and destruction of villus epithelium in the small intestine [34, 35]. To investigate whether KLF5 is expressed differently in these two settings, WT mice were given 8 or 15 Gy TBI to establish reparable and irreparable intestinal damage models. Small intestines were collected at different time points after irradiation for histopathological examination. H\&E staining of tissue sections confirmed that 8 Gy TBI induced reparable intestinal damage and 15 Gy TBI induced irreparable intestinal damage (Fig. S1).

Next, we measured KLF5 and its cellular localization in the small intestines of irradiated mice with Western blot and IHC. Western blot analysis indicated that KLF5 expression was induced in small intestines of 8 Gy-irradiated WT mice 3 days after exposure. KLF5 returned to baseline on day 7 (Fig. 1A). In 15 Gy-treated mice, intestinal KLF5 expression sharply decreased as early as 2 days post-TBI and recovered from day 4 (Fig. 1B).

The expression pattern of KLF5 revealed by IHC staining was consistent with the Western blotting results (Fig. 1C). In non-irradiated mice, KLF5 exhibited nuclear expression in proliferating epithelial cells at the base of the crypts in small intestines. At day 3 post 8 Gy TBI, the depth of the crypts increased noticeably and intense KLF5 staining was observed throughout the crypts. When the structure of the intestine had nearly returned to normal on day 7 , the expression of 
KLF5 was similar to that in the non-irradiated mice. However, in mice exposed to 15 Gy TBI, reduced crypt KLF5 was observed as early as 1 day after irradiation. When crypts disappeared from the intestinal mucosa on day 3, KLF5 expression was undetectable. On day 5, when aberrant regenerative crypts reemerged, KLF5 was strongly expressed in regenerative crypt epithelial cells. Thus, expression of KLF5 correlates with the progression of intestinal damage, suggesting an important role of KLF5 in radiation-induced intestinal injury.

In addition, analysis of serial sections revealed that KLF5 was expressed in Ki67-positive proliferative zones of the intestine, and in some cells that were positive for Musashi-1, a marker of intestinal stem cells (Fig. 1D).

A

NC $\quad 6 h \quad 1 d \quad 2 d \quad 3 d \quad 4 d \quad 5 d \quad 7 d$

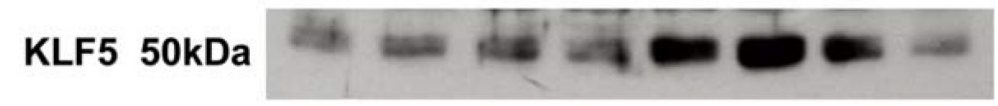

GAPDH 36kDa

B

NC $\quad 6 h \quad 12 h \quad 1 d \quad 2 d \quad 3 d \quad 4 d \quad 5 d$

KLF5 50kDa

GAPDH $36 \mathrm{kDa}$
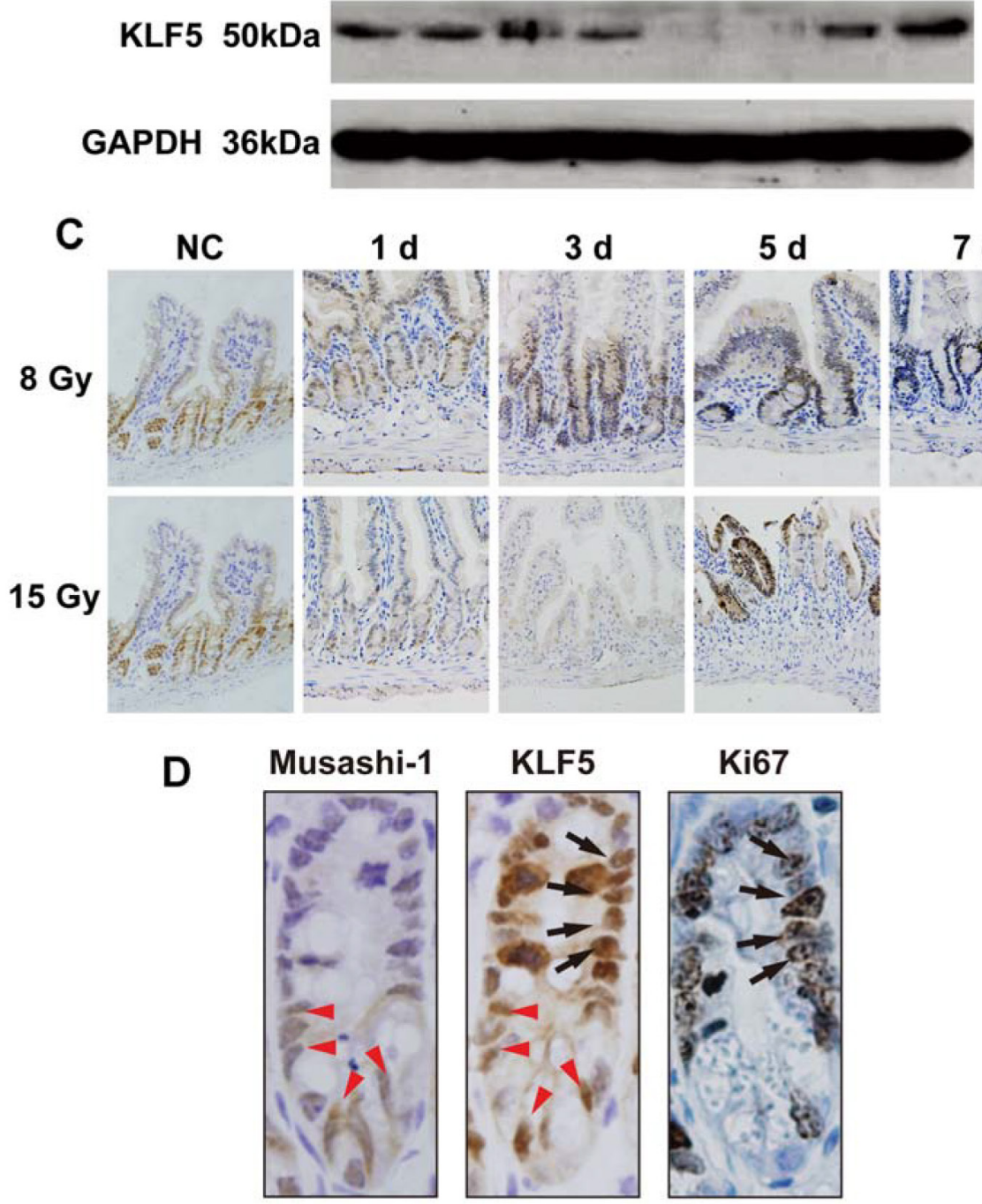

Fig 1. The levels and cellular localization of KLF5 in small intestine epithelia of WT mice after TBI. Animals were treated as depicted in Methods. Western blots of lysates from WT mice after 8 (A) or 15 Gy (B) TBI over 1 week. NC, non-irradiated control. For each time point, lysates were pooled from 3 separate mice. (C) Immunohistochemical staining of KLF5 in paraffin sections from small intestines of NC WT mice or those after 8 or 15 Gy TBI (magnification 200x). (D) Immunohistochemical analysis of sequential sections using antibodies against KLF5, Musashi-1 or Ki67 (magnification 1000x). (Black arrows indicate cells positive for KLF5 and Ki67; red arrowheads indicate cells positive for KLF5 and Musashi-1). 


\section{Histological analysis of increased intestinal damage in Vil-Cre; KIf5 $\mathrm{fl}^{\prime+}$ irradiated mice}

Mice with intestinal-specific deletion of KLF5 (Vil-Cre; Klf5f/fll mice) were created according to published methods [24] and theoretically ideal for examining the role of KLF5 in radiation-induced intestinal injury. However, approximately two-thirds of Vil-Cre; Klf5f/fll mice died within 2 days after birth and the remainder survived up to 8 weeks before dying due to significant weight loss and rectal prolapse. Therefore, mice with intestinal-specific knockdown of KLF5 (Vil-Cre; $\mathrm{Klf5} 5^{f /+}$ mice) were created and used in the following studies described here. Decreased KLF5 protein in knockdown animals was confirmed by IF (Fig. 2A) and Western blot (Fig. 2B). H\&E staining of ileal tissues revealed no obvious morphological changes in Vil-Cre; Klf5fl/+ mice compared to control mice (Fig. 3).

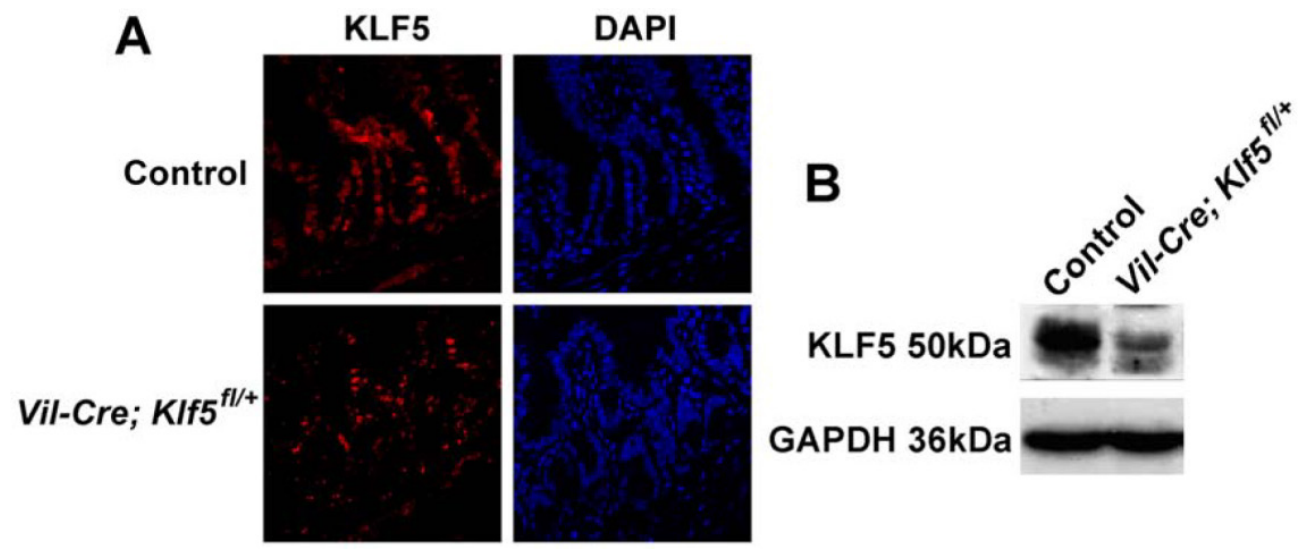

Fig 2. KLF5 expression is reduced in the small intestine epithelia of Vil-Cre; KIf5fl+ mice. (A) Immunofluorescent staining of KLF5 in small intestine sections from control and Vil-Cre; KIf5fl/+ mice (magnification $400 \times$ ). DAPI was used for nuclear counterstaining. (B) Detection of KLF5 levels in small intestines of control and Vil-Cre; KIf5fl/+ mice by Western blot, using pooled lysates from 3 mice per group.
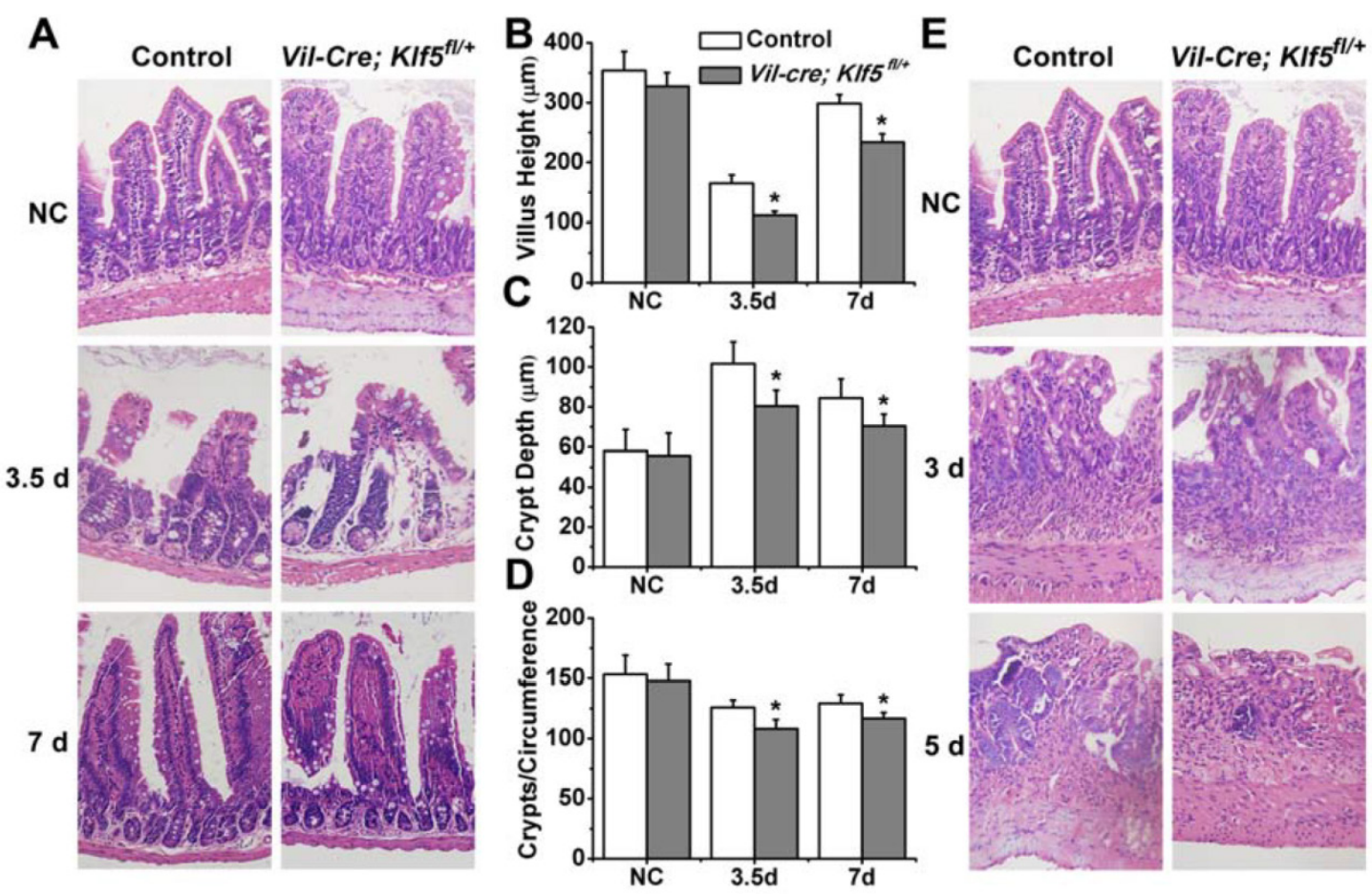

Fig 3. Vil-Cre; KIf5fll+ mice exhibit severe histological intestinal injuries after 8 or 15 Gy TBI. Representative H\&E staining of intestinal sections from control and Vil-Cre; KIf5fl/+ mice after 8 (A) or 15 Gy (E) TBI at indicated times (magnification 200x). NC, non-irradiated control. Bar graph of villus height (B), crypt depth (C) and crypts per circumference (D) determined by measuring vertically well-oriented crypt villus units from H\&E-stained sections of mice after 8 Gy TBI. For villus height or crypt depth measurement, at least 30 villi or crypts per mouse were measured. Crypts per circumference were counted in 3 separate tubular intestinal slices for each mouse. $* P<0.05 ; n=$ 
Vil-Cre; Klf5fl/+ and control mice were given 8 or 15 Gy TBI. At 3.5 days post 8 Gy TBI, villus heights of Vil-Cre; $\mathrm{Klf5} 5^{f /+}$ mice were reduced $32.3 \%$ compared to control mice $(P<0.05, \mathrm{n}=6)$ (Figs. $3 \mathrm{~A}$ and $\mathrm{B})$. Crypt depths were $20.9 \%$ lower in $\mathrm{Vil}-\mathrm{Cre}$; $\mathrm{Klf5} 5^{f /+}$ mice than in control mice $(P<0.05, \mathrm{n}=6)$ (Fig. 3C). Similar responses were observed when comparing crypts per circumference. There were $14.1 \%$ fewer crypts per circumference in $\mathrm{Vil}-\mathrm{Cre}$; $\mathrm{Klf5} \mathrm{fl}^{\mathrm{l}+}$ mice than in control mice $(P<0.05, \mathrm{n}=6)$ (Fig. 3D). On day 7 post-irradiation, although the intestinal mucosal morphology in both control and Vil-Cre; $\mathrm{Kl} / 5 \mathrm{ffl}^{\mathrm{f}}$ mice had almost recovered, there were still obvious differences between them. Villus heights, crypt depths, and crypts per circumference of $\mathrm{Vil}-\mathrm{Cre}$; $\mathrm{Klf5fl+}$ mice were reduced by $21.7 \%(P<0.05, \mathrm{n}=6), 16.6 \%(P<$ $0.05, \mathrm{n}=6)$ and $9.8 \%(P<0.05, \mathrm{n}=6)$, respectively, compared to control mice.

Destruction of villi and crypt disappearance occurred in both control and Vil-Cre; Klf5fl/t mice on day 3 post 15 Gy TBI (Fig. 3E). Aberrant regenerative crypts reemerged in intestinal mucosa of control mice but fewer crypts were observed in $\mathrm{Vil}-\mathrm{Cre}$; $\mathrm{Klf5} \mathrm{fl}^{\mathrm{l}+}$ mice at 5 days (Fig. 4F). Thus, Vil-Cre; $\mathrm{Klf} 5^{f /+}$ mice had more severe tissue damage and poorer recovery of the intestinal mucosa compared to control mice.

\section{Decreased epithelial proliferation in Vil-Cre; KIf5 $\mathrm{fll}^{\mathrm{+}}$ irradiated mice}

To determine whether KLF5 affects proliferation after irradiation, IHC for Ki67 was performed in intestinal tissues of control and Vil-Cre; $\mathrm{Klf5fl+}$ mice and Ki67-positive cells per crypt were counted. As shown in Fig. 4A, Ki67-positive cells increased in both control and Vil-Cre; $\mathrm{Klf5} 5^{f /+}$ mice at 3.5 days after 8 Gy TBI. Compared to the non-irradiated cohort, Ki67-positive cells per crypt increased by $105.9 \% \pm 19.8 \%$ in irradiated control mice, and Vil-Cre; $\mathrm{Klf5}^{f /+}$ mice had a smaller increase of $76.7 \% \pm 18.4 \%(P<0.05, \mathrm{n}=6)$ (Fig. $4 \mathrm{~B})$. IF analysis revealed that cells with more KLF5 expression had stronger Ki67 signals (Fig. 4C). In Vil-Cre; Klf5fl/+ mice, KLF5 was lower than in control mice, and there were fewer Ki67-positive cells per crypt (Fig. 4C). Thus, KLF5 and Ki67 expression are positively correlated.

At 6 h post 15 Gy TBI, Ki67 staining in Vil-Cre; $\mathrm{Kl} f 5^{f /+}$ mice was weaker than in control mice and Ki67-positive cells per crypt in Vil-Cre; $\mathrm{Klf5} / \mathrm{fl+}$ mice $(4.2 \pm 2.1)$ were significantly lower than in control mice $(9.4 \pm 2.3)(P<0.05, \mathrm{n}=6)$ (Fig. 4D). On day 3, some Ki67-positive cells remained in control mice where crypts had been located, but few Ki67-positive cells remained in the intestinal mucosa of Vil-Cre; $\mathrm{Klf5} \mathrm{fl}^{\mathrm{l}+}$ mice. At day 5, regenerative crypts that stained positive for Ki67 reemerged in control mice, but fewer regenerative crypts were observed in $\mathrm{Vil}-\mathrm{Cre}$; $\mathrm{Klf5} \mathrm{fl}^{\mathrm{l}+}$ mice $(P<0.05, \mathrm{n}=6$; Fig. 4E, F). Thus, KLF5 is indispensable for crypt regeneration after irreparable intestinal damage.

\section{Multiple signaling pathways are altered in re- sponse to down-regulation of KLF5 in the small intestine}

To understand the molecular response within the small intestine after down-regulation of KLF5, gene expression profiles of $\mathrm{Vil}-\mathrm{Cre} ; \mathrm{Klf5f/+}$ and control mice without TBI or $6 \mathrm{~h}$ post $15 \mathrm{~Gy}$ TBI were assessed with microarray. A total of 11,004 genes were expressed differentially (absolute fold change $\geq 2$ ) between non-irradiated control and $\mathrm{Vil}-\mathrm{Cre} ; \mathrm{Klf5}^{\mathrm{fl} /+}$ mice. Of these, 3,421 genes were up-regulated and 7,583 genes were down-regulated (Fig. 5A). At $6 \mathrm{~h}$ after 15 Gy TBI, 2,466 genes were differentially expressed between control and Vil-Cre; Klf5fl/+ mice, with 1,094 genes up-regulated and 1,372 down-regulated (Fig. 5A). The distribution of the differentially expressed genes, grouped according to fold-change, is shown in Table S2. Most differentially expressed genes fell into the 2- to 10-fold range.

Pathway analysis revealed that knockdown of Klf5 down-regulated genes associated with proliferation-related signaling networks such as MAPK, mTOR and PI3K/Akt pathways (Fig. 5B). Genes related to major signaling pathways in intestinal stem cells, such as Wnt, Hippo and Notch pathways, were also down-regulated in response to Klf5 knockdown.

We also noted that DNA damage repair-related genes were influenced by KLF5. Fig. 5B and C show that multiple DNA repair pathways were influenced by Klf5 knockdown. Genes related to the Fanconi anemia pathway (Fig. 6A), MMR (Fig. 6B) and NER (Fig. 6C) were down-regulated before irradiation, and at $6 \mathrm{~h}$ post 15 Gy TBI, genes involved in NHEJ (Fig. $6 \mathrm{D})$, a pathway that repairs DNA double-strand breaks, were down-regulated. Table S3 and Table S4 show differentially expressed genes involved in the Fanconi anemia pathway, MMR, NER and NHEJ. Expression patterns of 8 representative genes related to DNA damage repair (Ercc5, Culb4, Xpc, Revl, Lig1, Usp1, Nhej1 and Dcire1c) were assessed with qRT-PCR (Fig. 6E). Expression of all 8 genes was down-regulated, which is consistent with microarray assay data. Thus, KLF5 possibly promotes intestinal wound healing by regulating major signaling pathways associated with proliferation, intestinal stem cells, and DNA damage repair. 
A

Vil-Cre; KIff $5^{f l+}$
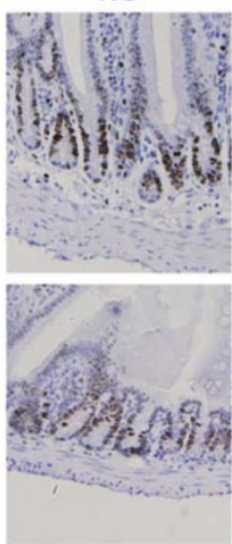

B

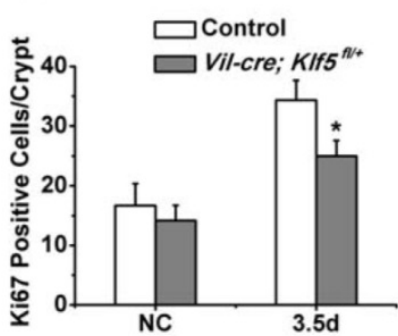

D
$6 h$

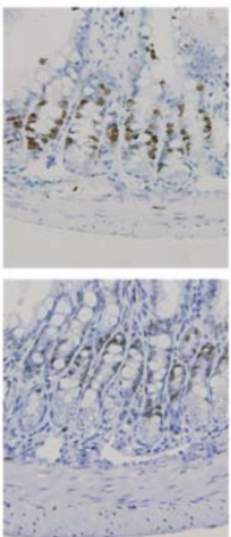

C

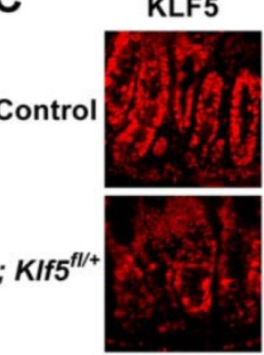

$6 h$

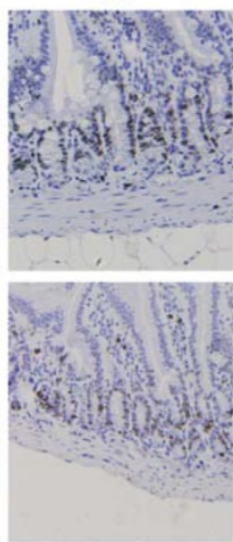

E

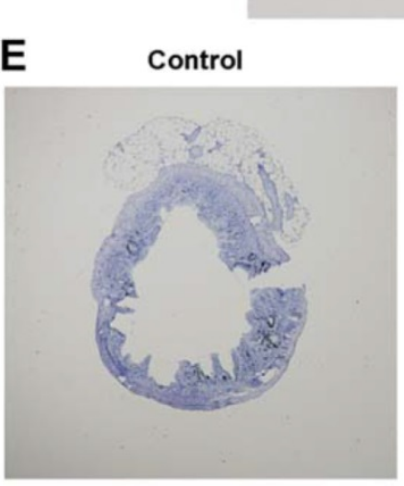

NC
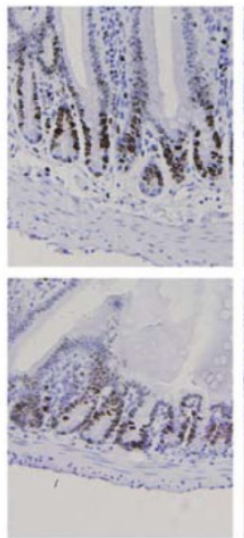

Vil-Cre; KIf5 ${ }^{f l+}$

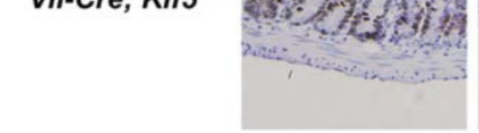

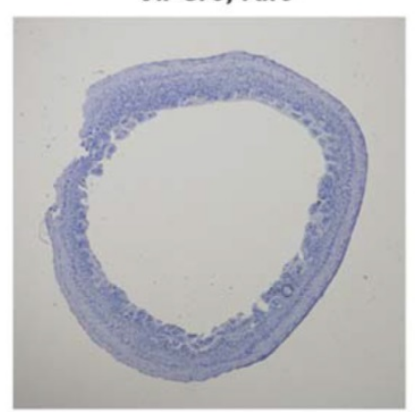

$3.5 \mathrm{~d}$
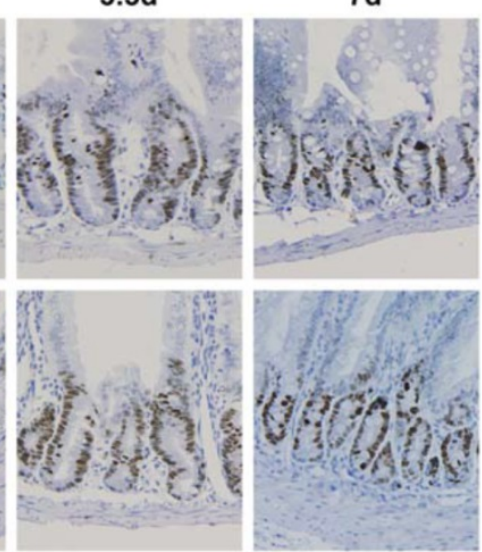

Ki67

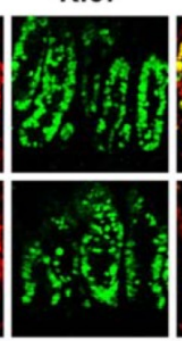

$3 d$

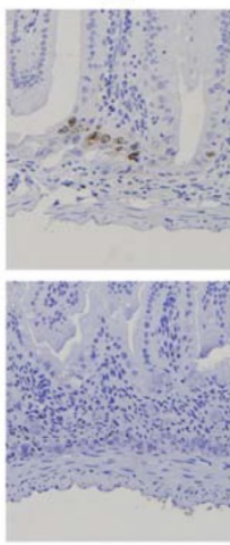

$\mathbf{F}$

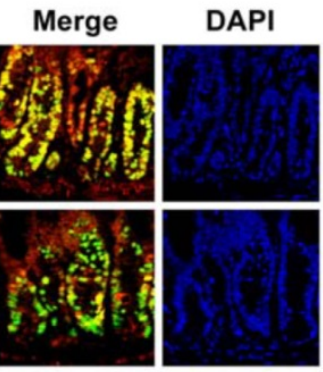

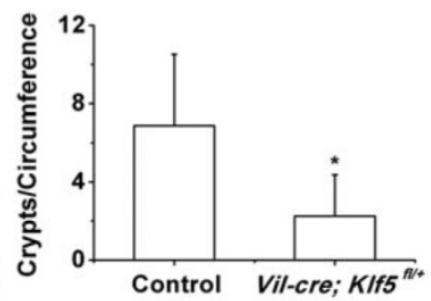

Fig 4. Proliferation is reduced in Vil-Cre; KIf5fl/+ mice compared to control cohorts after 8 or 15 Gy TBI. Intestinal tissues were treated as described in Methods after 8 (A) or 15 Gy (D) TBl and analyzed immunohistochemically for Ki67 expression (magnification 200x). NC, non-irradiated control. (B) Quantification of Ki67-positive cells per crypt determined from Ki67-immunostained sections of small intestines of control and Vil-Cre; KIfff//+ mice 3.5 days after 8 Gy TBI. At least 30 well-oriented crypts per mouse were counted. $* P<0.05 ; n=6$. (C) Cellular localization of Ki67 and KLF5 in small intestines of control and Vil-Cre; K/f5/l/+ mice at 3.5 days post 8 Gy TBI, as determined by confocal immunofluorescent staining (magnification 400x). Nuclear DNA (blue) was stained with DAPI. (E) Ki67 immunostaining in small intestines of control and Vil-Cre; KIf5fl/t mice at 5 days after $15 \mathrm{~Gy} \mathrm{TBI}$ (magnification 40x). (F) Quantification of crypts per circumference determined from Ki67-immunostained sections of small intestines at 5 days after 15 Gy TBI. Crypts were counted in 3 separate tubular intestinal slices per mouse. $* P<0.05 ; n=6$. 

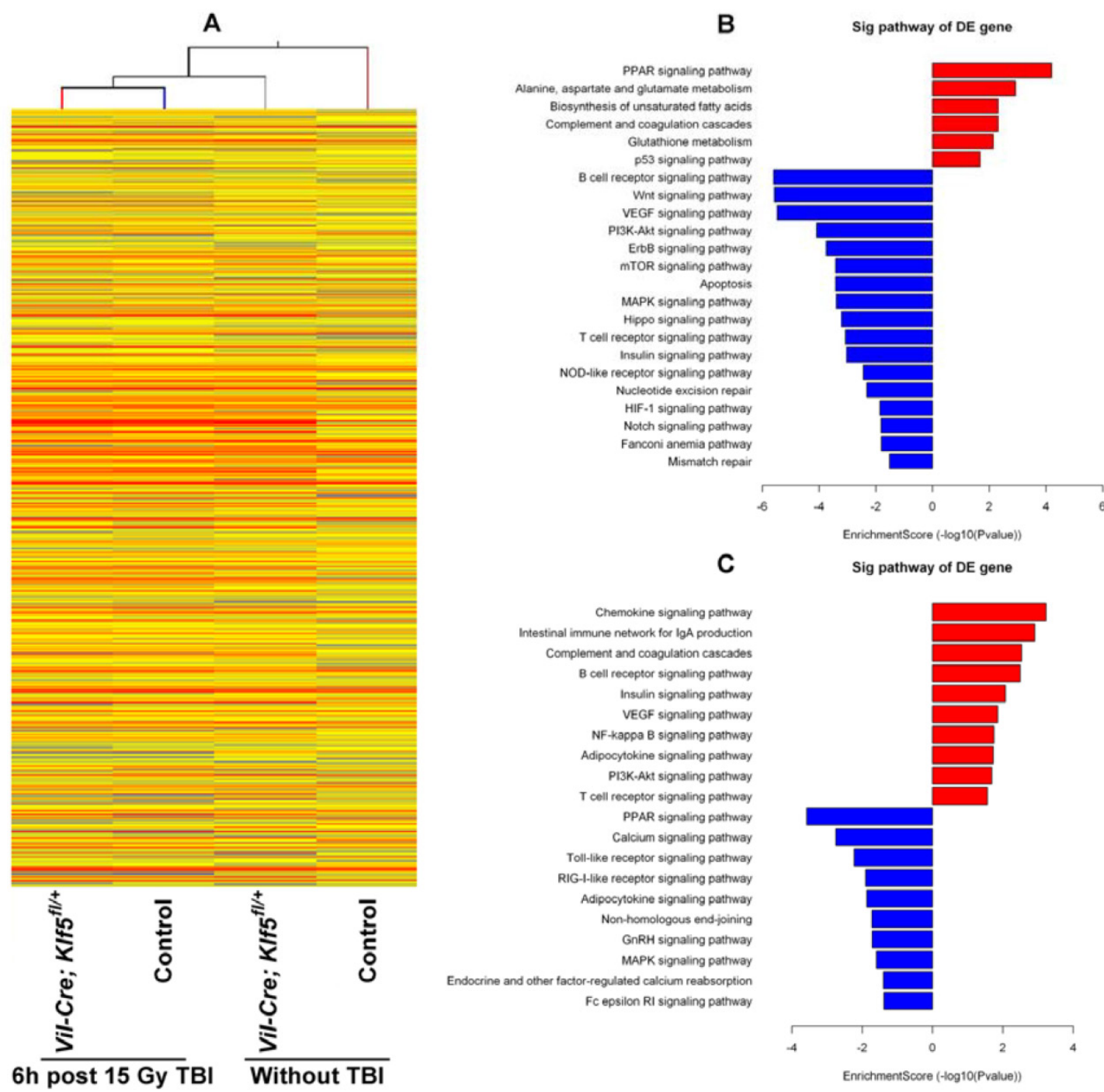

Fig 5. Significant pathways altered by down-regulation of KLF5 in the small intestine. (A) Clustering map of genes differentially expressed between Vil-Cre; Klf5fl/ and control mice without TBI or at $6 \mathrm{~h}$ post $15 \mathrm{~Gy}$ TBI. (B) Significant pathways altered in non-irradiated mice (Vil-Cre; Klf5fl/+ mice vs. control mice). (C) Significant pathways altered in mice $6 \mathrm{~h}$ post $15 \mathrm{~Gy}$ TBI (Vil-Cre; KIf5fl/+ mice vs. control mice). Red bars represent significant pathways for up-regulated genes. Blue bars represent significant pathways for down-regulated genes. DE, differentially expressed.

\section{Discussion}

The small intestinal epithelium is one of the most rapidly renewing mammalian tissues, renewing every 3-5 days. Thus, epithelial cells, especially crypt stem cells, are highly sensitive to radiation [36], which causes a deficient supply of intestinal epithelial cells, villus denudation, crypt atrophy or disappearance and mucosal architecture disruption. Little is known about the molecular mechanisms underlying intestinal damage and regeneration after exposure to ionizing radiation. Recent studies indicate that KLF5 is involved in protecting tissues from various injuries and promoting tissue restoration in response to external stresses such as bacterial pathogens [25] or chemical irritants [26]. Here, we report that KLF5 is induced in mouse intestinal tissues in instances of reversible damage induced by 8 Gy TBI, and elevated
KLF5 expression is concomitant with crypt regeneration. In contrast, after irreversible intestinal damage induced by 15 Gy exposure, expression of KLF5 is reduced in crypts prior to crypt disappearance. These results suggest that KLF5 expression correlates with the progression of intestinal damage. However, the mechanism responsible for opposing patterns of KLF5 expression in response to different doses of radiation needs further investigation.

Although we have determined that KLF5 expression was associated with the progression of intestinal damage, whether KLF5 has a predominant or subordinate role in this process is unclear. We observed that the intestinal tissue damage was more severe after irradiation in the Klf5 knockdown mouse model compared to control mice. In addition, fewer regenerating crypts were observed in intestinal mucosa of $\mathrm{Vil}-\mathrm{Cre}$; $\mathrm{Klf5}^{f /+}$ mice compared to control mice, 
especially after 15 Gy TBI. Thus, KLF5 is indispensable for crypt regeneration when the intestine has been irreparably damaged. Other studies with genetically modified mice suggest that loss of KLF5 in adult mouse intestinal tissues causes loss of crypt architecture and barrier function [24, 28, 37]. Our results underscore the important role of KLF5 in intestinal epithelial repair after irradiation, which may contribute to the etiology and treatment of radiation-induced intestinal damage.

Consistent with previous studies [25], KLF5 was expressed in Ki67-positive cells. We also found that KLF5 was expressed in Musashi-1-positive intestinal stem cells. Accumulating evidence suggested that KLF5 may be essential for self-renewal and maintenance of stemness in embryonic stem cells [38-41]. However, little is known about the functions of KLF5 in adult stem cell regulation. A recent study confirmed that inducible deletion of Klf5 in adult intes- tinal epithelium causes long-term loss of stem cell markers Lgr5, Ascl2 and Olf4 [28]. Another study suggested that inducible deletion of Klf5 in $\mathrm{Lgr5}^{+}$intestinal stem cells induces apoptosis in some stem and transit amplifying cells [23], indicating that KLF5 positively regulates intestinal stem cell survival but how this occurs is unclear. We found that KLF5 may regulate major signaling pathways in intestinal stem cells such as Wnt, Hippo and Notch pathways. Moreover, damage to stem cells is thought to be important for radiation-induced intestinal injury [42]. Hence, knockdown of KLF5 may exacerbate radiation-induced intestinal injury due to its effects on intestinal stem cells. Studies using mice with stem cell-specific inducible ablation of KLF5 will be required to determine whether a protective role of KLF5 in radiation-induced intestinal damage is mediated through intestinal stem cells.
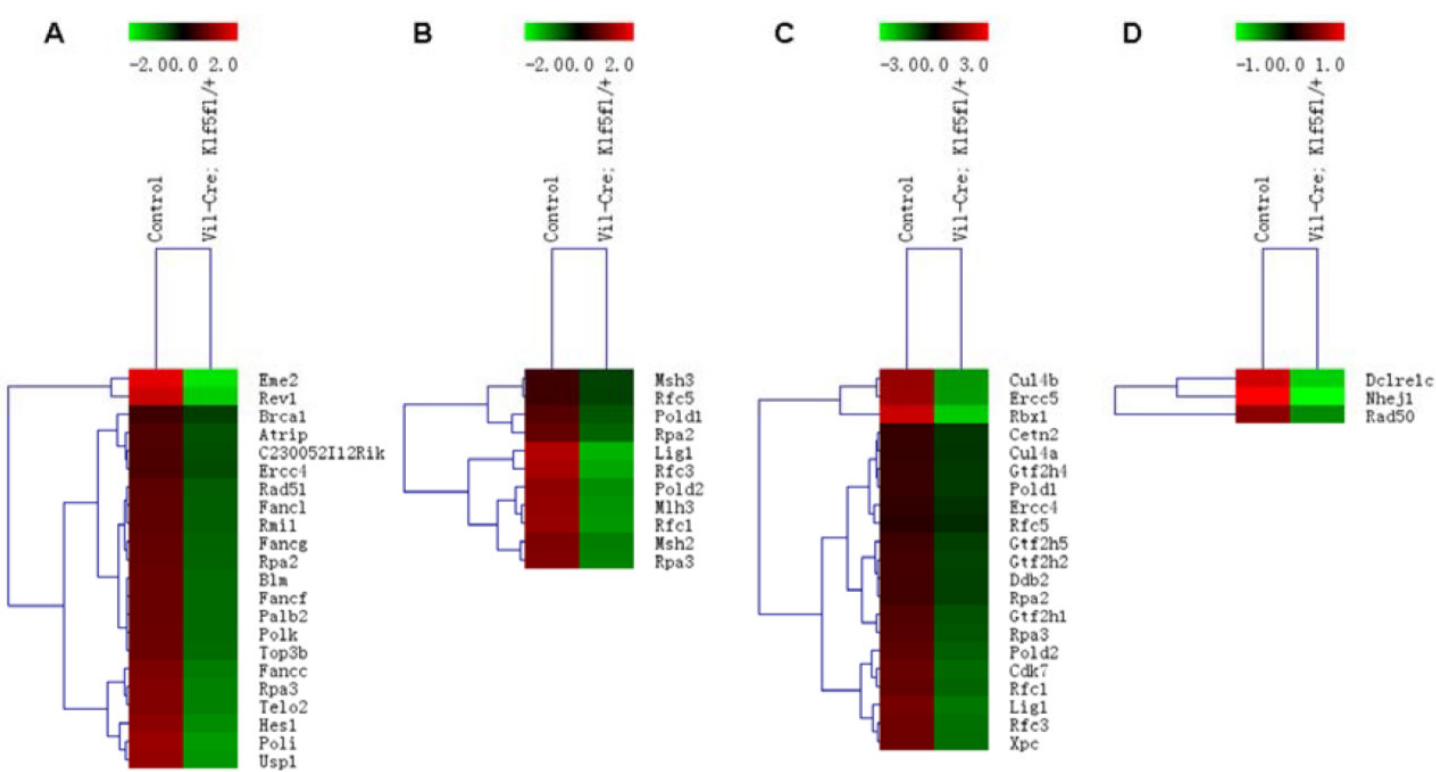

E

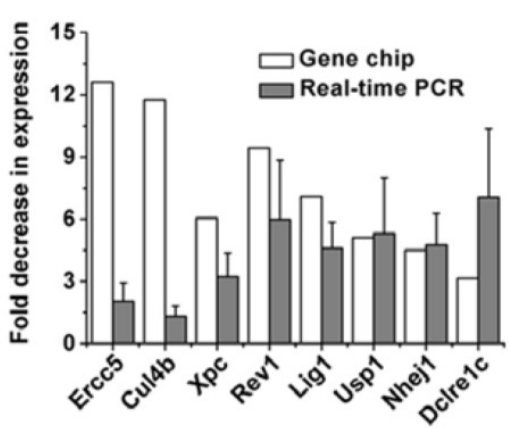

Fig 6. Heat maps and validation of differentially expressed genes related to DNA damage repair in response to knockdown of KLF5. (A) Fanconi anemia pathway genes in non-irradiated mice (Vil-Cre; Klf5fl/+ mice vs. control mice). (B) Mismatch repair genes in non-irradiated mice (Vil-Cre; KIf5fll+ mice vs. control mice). (C) Nucleotide excision repair genes in non-irradiated mice (Vil-Cre; KIf5fl/+ mice vs. control mice). (D) Non-homologous end-joining genes in mice $6 \mathrm{~h}$ post $15 \mathrm{~Gy}$ TBI (Vil-Cre; KIf5fl+ mice vs. control mice). (E) Quantitative real-time PCR validation for selected genes involved in DNA damage repair in control and Vil-Cre; KIf5fl/+ mice $(\mathrm{n}=3)$. 
DNA is the major target of ionizing radiation. A recent work indicated that double-strand DNA breaks (detected by $\gamma \mathrm{H} 2 \mathrm{AX}$ foci) exist extensively in both crypts and villi $1 \mathrm{~h}$ after 12 Gy TBI and are significantly reduced in crypts but remain high in villi $4 \mathrm{~h}$ after irradiation [43]. DNA damage repair after radiation-induced intestinal injury is less understood. Our work is the first to describe the effect of KLF5 on DNA damage repair pathways using high-throughput microarray analysis. In Klf5 knockdown mice, baseline expression of genes related to the Fanconi anemia pathway, MMR and NER were down-regulated in the absence of radiation, and NHEJ-related genes were down-regulated $6 \mathrm{~h}$ post $15 \mathrm{~Gy}$ TBI. Although the role of KLF5 in DNA damage repair has not been investigated, there is evidence that KLF4, which is of the same family as KLF5, is required for p53-mediated induction of p21 in response to DNA damage and inhibits p53 activation of the Bax promoter after gamma-radiation [44-46]. Considering that KLF4 and KLF5 bind to similar DNA sequences [47], KLF5 is likely to activate DNA damage repair-related genes through direct and/or indirect transcriptional cascades. Future studies will be focused on exploring the mechanisms of how KLF5 influences DNA damage repair.

In conclusion, KLF5 has a role in preventing intestinal injury induced by ionizing radiation by modulating DNA repair pathways. KLF5 signaling is a promising area of studies for identifying potential therapeutic targets for treating radiation-induced intestinal damage.

\section{Transcript Profiling}

Microarray data are available on the GEO database: accession number GSE66655.

\section{Supplementary Material}

Supplementary Methods, Figures and Tables.

http://www.ijbs.com/v11p1458s1.pdf

\section{Abbreviations}

ATM: ataxia telangiectasia mutated; BER: base excision repair; CBCs: crypt base columnar stem cells; DSS: dextran sulfate sodium; EGFR: epithelial growth factor receptor; FGF-BP: fibroblast growth factor binding protein 1; H\&E: hematoxylin and eosin; HR: homologous recombination repair; IF: Immunofluorescence assay; IHC: Immunohistochemical assay; IL-6: interleukin-6; KLF5: Krüppel-like factor 5; LPS: lipopolysaccharide; MMR: mismatch repair; mRNA: messenger RNA; NER: nucleotide excision repair; NHEJ: non-homologous end joining; PARP-1: poly ADP-ribose polymerase-1; PBS: phosphate-buffered saline; qRT-PCR: quantitative real-time polymerase chain reaction; TBI: total body irradiation; TNF-a: tumor necrosis factor-a; WT: wild type.

\section{Acknowledgment}

This work was supported by the National Natural Science Foundation of China (31300694), Jiangsu Provincial Natural Science Foundation (BK20130336), the National Defense Basic Scientific Research Program of China (B3820133001), the Priority Academic Program Development of Jiangsu Higher Education Institutions (PAPD) and Jiangsu Provincial Key Laboratory of Radiation Medicine and Protection.

\section{Competing Interests}

The authors have declared that no competing interest exists.

\section{References}

1. Potten CS. A comprehensive study of the radiobiological response of the murine (BDF1) small intestine. International journal of radiation biology. 1990; 58: $925-73$

2. Bismar MM, Sinicrope FA. Radiation enteritis. Current gastroenterology reports. 2002; 4: 361-5.

3. Williams JP, Brown SL, Georges GE, Hauer-Jensen M, Hill RP, Huser AK, et al. Animal models for medical countermeasures to radiation exposure. Radiation research. 2010; 173: 557-78.

4. Driak D, Osterreicher J, Vavrova J, Rehakova Z, Vilasova Z. Morphological changes of rat jejunum after whole body gamma-irradiation and their impact in biodosimetry. Physiological research / Academia Scientiarum Bohemoslovaca. 2008; 57: 475-9.

5. Somosy Z, Horvath G, Telbisz A, Rez G, Palfia Z. Morphological aspects of ionizing radiation response of small intestine. Micron. 2002; 33: 167-78.

6. Labejof LP, Galle P, Mangabeira PA, de Oliveira AH, Severo MI. Histological changes in rat duodenum mucosa after whole-body gamma irradiation. Cellular and molecular biology (Noisy-le-Grand, France). 2002; 48: 537-45.

7. Sterpone S, Cozzi R. Influence of XRCC1 Genetic Polymorphisms on Ionizing Radiation-Induced DNA Damage and Repair. Journal of nucleic acids. 2010; 2010.

8. Ch'ang HJ, Maj JG, Paris F, Xing HR, Zhang J, Truman JP, et al. ATM regulates target switching to escalating doses of radiation in the intestines. Nature medicine. 2005; 11: 484-90.

9. Clarke AR, Jones N, Pryde F, Adachi Y, Sansom OJ. 53BP1 deficiency in intestinal enterocytes does not alter the immediate response to ionizing radiation, but leads to increased nuclear area consistent with polyploidy. Oncogene. 2007; 26: 6349-55.

10. Ishizuka S, Martin K, Booth C, Potten CS, de Murcia G, Burkle A, et al. Poly(ADP-ribose) polymerase-1 is a survival factor for radiation-exposed intestinal epithelial stem cells in vivo. Nucleic acids research. 2003; 31: 6198-205.

11. Rotolo JA, Mesicek J, Maj J, Truman JP, Haimovitz-Friedman A, Kolesnick R, et al. Regulation of ceramide synthase-mediated crypt epithelium apoptosis by DNA damage repair enzymes. Cancer research. 2010; 70: 957-67.

12. Toft NJ, Winton DJ, Kelly J, Howard LA, Dekker M, te Riele H, et al. Msh2 status modulates both apoptosis and mutation frequency in the murine small intestine. Proceedings of the National Academy of Sciences of the United States of America. 1999; 96: 3911-5.

13. Sansom OJ, Bishop SM, Court H, Dudley S, Liskay RM, Clarke AR. Apoptosis and mutation in the murine small intestine: loss of Mlh1- and Pms2-dependent apoptosis leads to increased mutation in vivo. DNA repair. 2003; 2 : 1029-39.

14. Hua G, Thin TH, Feldman R, Haimovitz-Friedman A, Clevers H, Fuks Z, et al. Crypt base columnar stem cells in small intestines of mice are radioresistant. Gastroenterology. 2012; 143: 1266-76.

15. Sogawa K, Imataka H, Yamasaki Y, Kusume H, Abe H, Fujii-Kuriyama Y. cDNA cloning and transcriptional properties of a novel GC box-binding protein, BTEB2. Nucleic acids research. 1993; 21: 1527-32.

16. Nandan MO, Yoon HS, Zhao W, Ouko LA, Chanchevalap S, Yang VW. Kruppel-like factor 5 mediates the transforming activity of oncogenic H-Ras. Oncogene. 2004; 23: 3404-13.

17. Nandan $\mathrm{MO}$, Chanchevalap S, Dalton WB, Yang VW. Kruppel-like factor 5 promotes mitosis by activating the cyclin $\mathrm{B} 1 / \mathrm{Cdc} 2$ complex during oncogenic Ras-mediated transformation. FEBS letters. 2005; 579: 4757-62.

18. Liu R, Zheng HQ, Zhou Z, Dong JT, Chen C. KLF5 promotes breast cell survival partially through fibroblast growth factor-binding protein 1-pERK-mediated dual specificity MKP-1 protein phosphorylation and stabilization. The Journal of biological chemistry. 2009; 284: 16791-8. 
19. Yang Y, Goldstein BG, Nakagawa H, Katz JP. Kruppel-like factor 5 activates MEK/ERK signaling via EGFR in primary squamous epithelial cells. Faseb J. 2007; 21: 543-50.

20. Zhu N, Gu L, Findley HW, Chen C, Dong JT, Yang L, et al. KLF5 Interacts with p53 in regulating survivin expression in acute lymphoblastic leukemia. The Journal of biological chemistry. 2006; 281: 14711-8.

21. Dong JT, Chen C. Essential role of KLF5 transcription factor in cell proliferation and differentiation and its implications for human diseases. Cell Mol Life Sci. 2009; 66: 2691-706

22. McConnell BB, Ghaleb AM, Nandan MO, Yang VW. The diverse functions of Kruppel-like factors 4 and 5 in epithelial biology and pathobiology. Bioessays. 2007; 29: 549-57.

23. McConnell BB, Kim SS, Yu K, Ghaleb AM, Takeda N, Manabe I, et al. Kruppel-like factor 5 is important for maintenance of crypt architecture and barrier function in mouse intestine. Gastroenterology. 2011; 141: 1302-13, 13 e1-6.

24. Nakaya T, Ogawa S, Manabe I, Tanaka M, Sanada M, Sato T, et al. KLF5 regulates the integrity and oncogenicity of intestinal stem cells. Cancer research. 2014; 74: 2882-91.

25. McConnell BB, Klapproth JM, Sasaki M, Nandan MO, Yang VW. Kruppel-like factor 5 mediates transmissible murine colonic hyperplasia caused by Citrobacter rodentium infection. Gastroenterology. 2008; 134: 1007-16.

26. McConnell BB, Kim SS, Bialkowska AB, Yu K, Sitaraman SV, Yang VW. Kruppel-like factor 5 protects against dextran sulfate sodium-induced colonic injury in mice by promoting epithelial repair. Gastroenterology. 2011; 140: $540-9$ e2.

27. Tetreault MP, Alrabaa R, McGeehan M, Katz JP. Kruppel-like factor 5 protects against murine colitis and activates JAK-STAT signaling in vivo. PloS one. 2012; 7: e38338.

28. Bell KN, Shroyer NF. Krupple-like factor 5 is required for proper maintenance of adult intestinal crypt cellular proliferation. Digestive diseases and sciences. 2015; 60: 86-100.

29. Chen C, Benjamin MS, Sun X, Otto KB, Guo P, Dong XY, et al. KLF5 promotes cell proliferation and tumorigenesis through gene regulation and the TSU-Pr1 human bladder cancer cell line. International journal of cancer. 2006; 118: 1346-55.

30. Nandan MO, McConnell BB, Ghaleb AM, Bialkowska AB, Sheng H, Shao J, et al. Kruppel-like factor 5 mediates cellular transformation during oncogenic KRAS-induced intestinal tumorigenesis. Gastroenterology. 2008; 134: 120-30.

31. Zhao Y, Hamza MS, Leong HS, Lim CB, Pan YF, Cheung E, et al. Kruppel-like factor 5 modulates p53-independent apoptosis through Pim1 survival kinase in cancer cells. Oncogene. 2008; 27: 1-8.

32. Chanchevalap S, Nandan MO, McConnell BB, Charrier L, Merlin D, Katz JP, et al. Kruppel-like factor 5 is an important mediator for lipopolysaccharide-induced proinflammatory response in intestinal epithelial cells. Nucleic acids research. 2006; 34: 1216-23.

33. Li M, Ma Y, Huang P, Du A, Yang X, Zhang S, et al. Lentiviral DDX46 knockdown inhibits growth and induces apoptosis in human colorectal cancer cells. Gene. 2015; 560: 237-44.

34. De Both NJ, Vermey M. Epithelial regeneration of transposed intestine after high doses of X-irradiation. International journal of radiation biology and related studies in physics, chemistry, and medicine. 1976; 29: 17-26.

35. Potten CS. Radiation, the ideal cytotoxic agent for studying the cell biology of tissues such as the small intestine. Radiation research. 2004; 161: 123-36.

36. Harfouche G, Martin MT. Response of normal stem cells to ionizing radiation: a balance between homeostasis and genomic stability. Mutation research. 2010; 704: 167-74.

37. Nandan MO, Ghaleb AM, Liu Y, Bialkowska AB, McConnell BB, Shroyer KR, et al. Inducible intestine-specific deletion of Kruppel-like factor 5 is characterized by a regenerative response in adult mouse colon. Developmental biology. 2014; 387: 191-202

38. Ema $M$, Mori $D$, Niwa $H$, Hasegawa $Y$, Yamanaka $Y$, Hitoshi $S$, et al Kruppel-like factor 5 is essential for blastocyst development and the normal self-renewal of mouse ESCs. Cell stem cell. 2008; 3: 555-67.

39. Jiang J, Chan YS, Loh YH, Cai J, Tong GQ, Lim CA, et al. A core Klf circuitry regulates self-renewal of embryonic stem cells. Nature cell biology. 2008; 10: 353-60.

40. Parisi S, Passaro F, Aloia L, Manabe I, Nagai R, Pastore L, et al. Klf5 is involved in self-renewal of mouse embryonic stem cells. Journal of cell science. 2008; 121: 2629-34.

41. Parisi S, Russo T. Regulatory role of Klf5 in early mouse development and in embryonic stem cells. Vitamins and hormones. 2011; 87: 381-97.

42. Yu J. Intestinal stem cell injury and protection during cancer therapy. Translational cancer research. 2013; 2: 384-96.

43. Wang F, Cheng J, Liu D, Sun H, Zhao J, Wang J, et al. P53-participated cellular and molecular responses to irradiation are cell differentiation-determined in murine intestinal epithelium. Archives of biochemistry and biophysics. 2014; 542: 21-7.

44. Zhou Q, Hong Y, Zhan Q, Shen Y, Liu Z. Role for Kruppel-like factor 4 in determining the outcome of $\mathrm{p} 53$ response to DNA damage. Cancer research. 2009; 69: 8284-92.

45. Zhang W, Geiman DE, Shields JM, Dang DT, Mahatan CS, Kaestner KH, et al. The gut-enriched Kruppel-like factor (Kruppel-like factor 4) mediates the transactivating effect of $\mathrm{p} 53$ on the p21WAF1/Cip1 promoter. The Journal of biological chemistry. 2000; 275: 18391-8.
46. Ghaleb AM, Katz JP, Kaestner KH, Du JX, Yang VW. Kruppel-like factor 4 exhibits antiapoptotic activity following gamma-radiation-induced DNA damage. Oncogene. 2007; 26: 2365-73.

47. Dang DT, Zhao W, Mahatan CS, Geiman DE, Yang VW. Opposing effects of Kruppel-like factor 4 (gut-enriched Kruppel-like factor) and Kruppel-like factor 5 (intestinal-enriched Kruppel-like factor) on the promoter of the Kruppel-like factor 4 gene. Nucleic acids research. 2002; 30: 2736-41. 